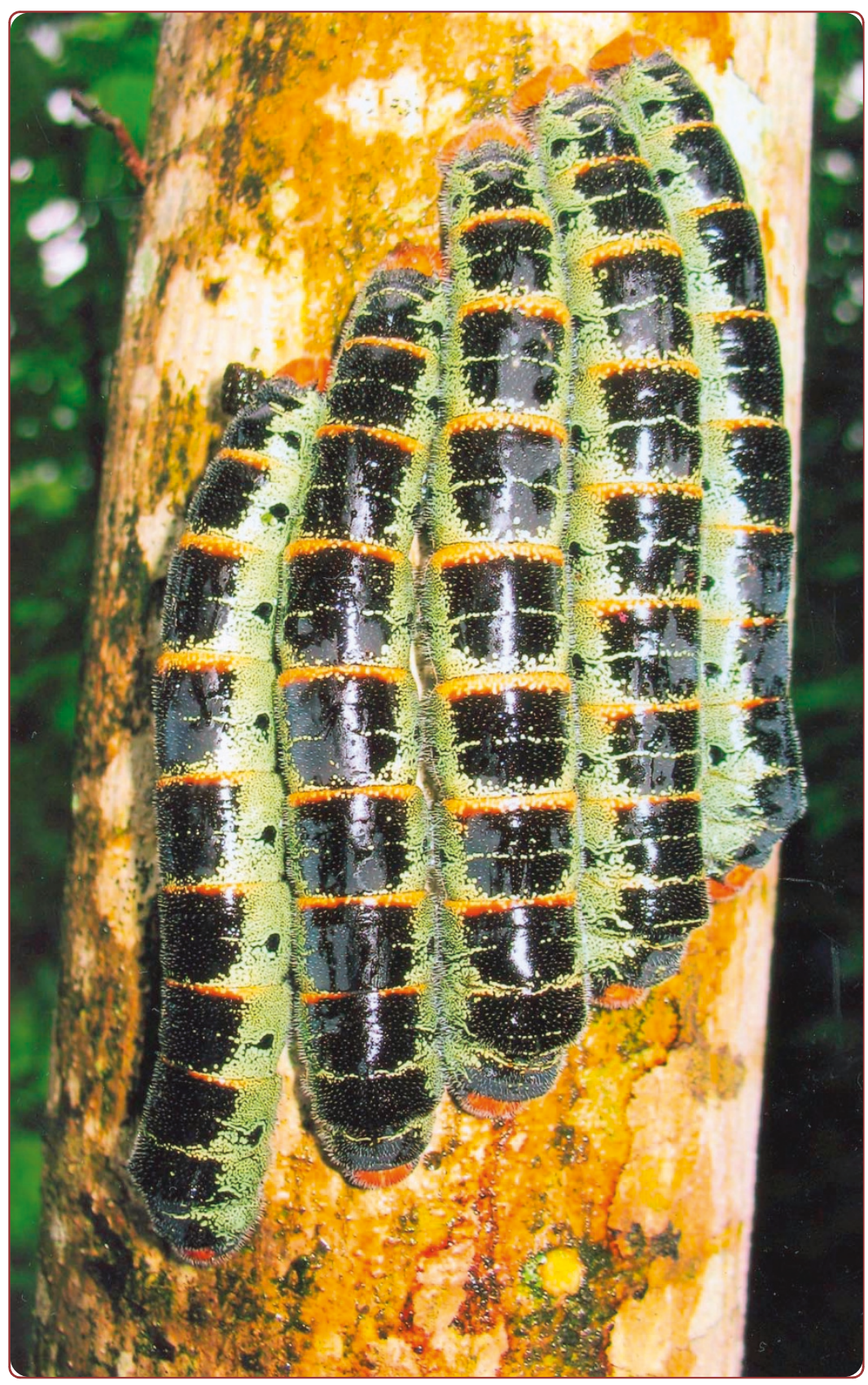

\title{
Edible Lepidoptera in Mexico: Geographic distribution, ethnicity, economic and nutritional importance for rural people
}

Ramos-Elorduy et al. 


\title{
Edible Lepidoptera in Mexico: Geographic distribution, ethnicity, economic and nutritional importance for rural people
}

\author{
Julieta Ramos-Elorduy ${ }^{1 *}$, José MP Moreno', Adolfo I Vázquez', Ivonne Landero², Héctor Oliva-Rivera², \\ Víctor HM Camacho'
}

\begin{abstract}
In this paper, we reported the butterflies and moths that are consumed in Mexico. We identified 67 species of Lepidoptera that are eaten principally in their larval stage in 17 states of Mexico. These species belong to 16 families: Arctiidae, Bombycidae, Castniidae, Cossidae, Geometridae, Hepialidae, Hesperiidae, Lasiocampidae, Noctuidae, Nymphalidae, Papilionidae, Pieridae, Pyralidae, Saturniidae, Sesiidae, and Sphingidae.

Saturniidae, Pieridae, Noctuidae and Nymphalidae were the more species consumed with 16, 11, 9, and 8 species, respectively.

The genera with the largest numbers of species were: Phassus, Phoebis, Hylesia and Spodoptera, with three species. Their local distribution, corresponding to each state of Mexico, is also presented.
\end{abstract}

\section{Background}

Lepidoptera is one of the richest Insecta orders. Their larvae serve as food for many ethnic groups around the world [1,2]; they are often prepared charcoaled in salty water or, in some cases, fried or mixed with other food [3]. Also contribute a great amount of energy and protein to indigenous diet [4]. In general, this reflects their availability. In the forests of the Central African Republic, some species are so abundant, that when they are in the last larval stage, their excrement fall sounding like heavy raindrops, and two months later, the soil becomes white due to the mycelium that develops [Ramos-Elorduy J, Personal observations, 1990].

The inhabitants make good use of them, storing and selling. This help the people to obtain income that is necessary in a subsistence economy. Ancient Mexicans were traded larvae of Pantherodes pardalaria and Aegiale hesperiaris [5]. Peasants know very well when and where is the biggest and tasty larval stage. People even make long journeys to obtain them; however, because of an over-exploitation, as in Zambia happened,

\footnotetext{
* Correspondence: relorduy@ibunam2.ibiologia.unam.mx

'Instituto de Biología, UNAM, Apartado Postal 70-153, 04510, México DF, México

Full list of author information is available at the end of the article
}

establish a law to enforce a closed season, to prevent extinction of Gynanisa maja and Gonimbrasia belina named "mumpa" [6], exploiting it in a rational way to balance preservation and exploitation [7].

The use of insects as food by the different ethnia of Mexico is a very complete study at Mexico that achieve 549 species [8]. We have documented 14 orders of the Insecta Class, including Lepidoptera.

A study of the edible species of Lepidoptera in Mexico has not yet been accomplished.

\section{Methods}

\section{Field}

Field work was conducted in 17 states of Mexico, including in 235 localities in: Chiapas (16), Chihuahua (2), Distrito Federal (22), Durango (1), Guanajuato (2) Guerrero (8), Hidalgo (64) State of México (51), Michoacán (5), Oaxaca (16), Puebla (17), Querétaro (1), Quintana Roo (2), Tlaxcala (15), Veracruz (10), Yucatán (1) and Zacatecas (2).

Emic-type interviews with an ethicist focus took place [9]; meetings were in rural areas, small towns, villages and cities. Their goal was to investigate the tracking, gathering, fixing and commercialization.

\section{() Biomed Central}


For collected, we use aerial nets, paint-brushes, knives or "machetes" and some by hand.

The larvae and pupal stages were placed in $70 \%$ alcohol solution or on dry ice if they were intended for chemical analysis. Adults were placed in potassium cyanide with plaster and then put in glassed paper envelopes labeled with the data.

\section{Laboratory}

For identification, adults were placed in a humid camera and mounted; after labeled, identified and catalogued. Forward were placed in the National Collection of Edible Insects of Mexico kept in the Institute of Biology, UNAM. The immature stages were placed in Khale Liquid for preservation. For identification, keys were used [10-17]. Our determinations were ratified by several specialists. With this information, the corresponding tables were elaborated.

The identification of hosts and the ecosystems was accomplished using different sources: De Vries [18], Martínez [19] and Rzedowski [20].

\section{Results and Discussion}

\section{Diversity and ethnicity}

We identified 67 species of Lepidoptera as being eaten in Mexico, in Table 1 shows family, subfamily, scientific name, places of consumption, developmental stage or stages consumed, common name, principal ethnia that use them as food, hosts, and principal ecosystems where they were localized.

The 13 families are in decreasing order of species number: Saturnidae (16), Pieridae (11), Noctuidae (9), Nymphalidae (8), Sphingidae (4), Arctiidae (4), Hepialidae (3), Hesperidae, Papilionidae and Geometridae (2) each one, Cossidae, Pyralidae, Sesiidae, Castniidae, Bombycidae, and Lasiocampidae (1) each one (Table 2, Figure 1).

The species number in each genus is indicated in figure 2 . It can be seen that most of the genera have only one species included (68.75\%), followed by the bispecific $(18.75 \%)$ and at the end trispecific genera (12.5\%).

The most represented genera were Phassus, Phoebis, Hylesia, and Spodoptera (Table 3).

Lepidoptera are eaten in $85.41 \%$ as larvae, $8.33 \%$ as larvae and pupae and in $6.25 \%$ as adults.

We found 29 ethnic groups that consume Lepidoptera in Mexico: Amuzgo, Chatinos, Chinantecos, Cholos, Huasteco, Huaves, Lacandones, Matlazinca, Maya, Mazahua, Mazatecas, Mixes, Mixtec, Nahuatl, Otomi, Otopame, Popolucas, Tarahumara, Tarascan, Tepehuano, Tlapaneco, Totonaco, Tojolabal, Triques, Tzeltal, Tzotzil, Yutoazteca, Zapotec and Zoques.

\section{Geographic Distribution}

These Lepidoptera species were found in those states of the central, south and southeast regions of the country. The highest number of species (22) was recorded in the eastern part of Veracruz, followed by Hidalgo (17), Distrito Federal (the capital) (16), and Chiapas and Puebla (12 species each). The remaining states, each one had six or fewer edible species.

With regard to the ecosystems [20], these species are attached from the pine oak forest, to the savannah and palmar. The Lepidoptera are also present in several agronomic plants, such as maize, alfalfa, cabbage and cauliflower, depending on the species.

\section{Anthropolarvifagia of Lepidoptera in the World}

Bergier [21] reports 15 species for the world, for America only one species Hesperiaris sp., in two countries. Taylor [22] registered 25 species in 12 families. Silow [23] describes 42 species of the genera Gonimbrasia, Imbrasia, Bunaea, Bunaeopsis, Cirina, Pseudantheraea, Micragone, Olocerina, and Melanocera, 33 of them are eaten in Zambia. It is important to mention that all these authors only did bibliographic research. In contrast, Malaisse and Parent [24] performed long-term field work studying Meridian Shaba area in Republic of Congo and in Zambia reporting 37 species (70\% classified) and Latham [25] in Low-Congo, documented 31 species (77\% classified). In both studies, the principal families were Attacidae and Notodontidae. Banjo et al. [26] reported six species in Nigeria, four of Anaphe genus. Oliveira et al. [27] also reported four species eaten in Angola. We note that six references are books and almost all refer to Africa.

Wen [28] in China presented 66 species, 20 genera and 17 families; 36 species of Hepialus genus. Mitsuhashi [29] reported five species in Japan.

Paoletti et al. [30] noted that larvae of Castniidae, Noctuidae and Sphingidae families are consumed in the Amazon area. Our report has 67 species occurring in just a part of the country.

\section{Rural Nutritional Importance}

For rural peasants, the big diversity that Edible Lepidoptera has, besides the good nutritive value achieve (18-57\% proteins, $7-77 \%$ fats, $0.7-8 \%$ minerals, $0.8-25 \%$ carbohydrates and 3-29\% crude fiber, $231-777 \mathrm{kcal} / 100$ g, [4], and their good flavor that gives their fats, united to the abundance of their populations, conspicuity of their specimens (latest larval stage) that save various important nutrients as proteins and the numerous muscles they posses, combined with their quick preparation (only roasted or boiled), and their innocuity, the easiness to store, make of them an item very searched plus their versability of fix make the Lepidopterans a suitable food, 
Table 1 TAXONOMY

\begin{tabular}{|c|c|}
\hline \multicolumn{2}{|c|}{ FAMILY HEPIALIDAE } \\
\hline \multicolumn{2}{|c|}{$\begin{array}{l}\text { 1.- Phassus trajesa } \\
\text { Schaus. }\end{array}$} \\
\hline Places: & Argovia, Independencia, Ixtapa, Chiapas. \\
\hline Edible stage: & larvae. \\
\hline Common names: & gusanillo (Esp), gusano tindáco (Zap), gusano yutu lolo (Mix). \\
\hline Ethnos: & Maya, tzotzil, tzeltal, chol, lacandon, tojolabal. \\
\hline Host: & Buddleia americana L., Senecio salignus D.C. \\
\hline Ecosystems. & Pine-oak forest, Tropical decidous forest, Arid tropical scrub, Cloud forest, Rain forest. \\
\hline \multicolumn{2}{|c|}{ 2.- Phassus triangularis Edwards. 1885} \\
\hline Places: & $\begin{array}{l}\text { Santa Ana Tlacotenco, San Pablo Oztotepec, San Salvador Cuahtenco, San Pedro Atocpan, San Bartolo } \\
\text { Xicomulco, San Antonio Tecomitl, Milpa Alta, DF. San Rafael, Pueblo Nuevo, Amanalco de Becerra, } \\
\text { Tenancingo, Tequixquiac, Valle de Bravo, (Mex). Yosotato, Coatzospan, Jamiltepec, Oaxaca. Necoxtla, } \\
\text { Zongolica, Veracruz. }\end{array}$ \\
\hline Edible stage: & larvae. \\
\hline Common names: & Gusanillo (Esp), gusano rayado (Esp), gusano grande (Esp), nduyacacitl (Mix), gusano gordo de la jarilla (Esp), chiáhuitl (Ntl). \\
\hline Ethnos: & $\begin{array}{l}\text { Yutoazteca, Nàhuatl, Otomí, Otopame, Mazahua, Matlazinca, Zapoteco, Mixteco, Mixe, Popoluca, Chatinos, Chinantecos, } \\
\text { Mazatecos, Zoques, Triques, Huave, Totonaca, Huasteco. }\end{array}$ \\
\hline Host: & Buddleia parviflora H.B.K., Senecio salignus D.C. \\
\hline Ecosystems: & Arid tropical scrub, Rain forest. \\
\hline \multicolumn{2}{|l|}{ 3.- Phassus sp. } \\
\hline Places: & $\begin{array}{l}\text { San Bartolo Xicomulco, San Pedro Atocpan, Milpa Alta, DF. Jilotepec, Cuautitlán de Romero Rubio, Villa del Carbón, San } \\
\text { Rafael, Pueblo Nuevo Mex. Santo Tomás, Tecomulco, Carpinteros, Atlapexco, Durango, Huasca, Hueyapán, Hidalgo. } \\
\text { Cañada, Coatzospan, Jamiltepec, San Juan Coatzalapan, Yosotato Oaxaca. Santiago Yancuitlalpan, Cuetzalan, Alchichica, } \\
\text { Puebla. Felipe Carrillo Puerto, Quintana Roo. San Pablo del Monte, Xicoténcatl, Xaltocan, Tetla, Tlaxcala. Chocamán, } \\
\text { Veracruz. }\end{array}$ \\
\hline Edible stage: & larvae. \\
\hline Common name: & gusanillo (Esp), gusano del aile (Esp), gusano del Tepozán (Xoc), calpulocuillin (Ntl). \\
\hline Ethnos: & $\begin{array}{l}\text { Yutoazteca, Nàhuatl, Otomí, Otopame, Mazahua, Matlazinca, Mixe, Popoluca, Chatinos, Chinantecos, } \\
\text { Mazatecos, Zoques, Triques, Huave. }\end{array}$ \\
\hline Host: & Senecio salignus D.C. \\
\hline Ecosystems. & Arid tropical scrub, Rain forest. \\
\hline
\end{tabular}

FAMILY COSSIDAE

4.- Subfamily: Chilecomadiinae

Comadia redtenbacheri Hammerschmidt 1848 (Figure 3)

Places:

San Pedro Atocpan, San Salvador Cuahtenco, San Jerónimo Miacatlán, Santa Ana Tlacotenco, San Bartolo Xicomulco, San Lorenzo Tlacoyucan, San Agustín Ohtenco, San Pablo Oztotepec, San Antonio Tecomitl, San Francisco Tecoxpa, San Juan Tepenahuac, Milpa Alta, DF. San Bartolo Morelos, Santiago Tianguistenco, Almoloya de Juárez, Villa Nicolás Romero, Oxtotipac, Lomas de Guadalupe, Cuautitlán Izcalli, Cuautitlán de Romero Rubio, San Juan Zitlaltepetl, Villa del Carbón, Santiago Tilapa, Almoloya del Río, Atlacomulco, Ixtlahuaca, Jalatlaco, Zumpango, Ozumba, San Pablo Jalalpan, Toluca, Mex. Valle de Santiago, Guanajuato. Venta de Guadalupe, Pachuquilla, Pinalito, Pozuelos, San Miguel Regla, Tlaxcoapan, Tulancalco, Trancas, Molango, Tepetitlán. Tulancingo, Zimapán, Cieneguillas, Durango, El Cajón, Ismolintla, El Dexthi, San Juanico, Ixmiquilpan, Pachuca, Tula de Allende, Jacala, San Sebastián Jonacapa, Tinaco, Tezontepec, Santa Ana Bertha, Chapantongo, Atotonilco de Tula, Maravillas, Hueyapán, Singuilucan, Santo Tomás, Cuautepec, Texcaltepec, Chilcuautla, Xochitlán, Venustiano Carranza, Actopan, Valle del Mezquital, Ajacuba, Apan, Atotonilco el Grande, Huichapán, Mixquihuala, San Nicolás Atexcoco, San Antonio Sabanillas, Singuilucan, Hidalgo. San Pedro Tarímbaro, Michoacán Vigastepec, Santiago Apoala Santa María Nduayaco, Tlacolula, Ocotlán, Oaxaca. Tehuacán, Chapulco, Acatlán de Osorio, Puebla. San Juan del Río, Querétaro. Calpulalpan, Cuapixtla, Huamantla, Ixtacuixtla, San Pablo del Monte, Tetla, Totolac, Xicoténcatl, Apizaco, San Pablo Matamoros, Tlaxcala. Jalpa, Zacatecas, Perote, Naolinco, Veracruz.

Edible stage larvae. 
Table 1 TAXONOMY (Continued)

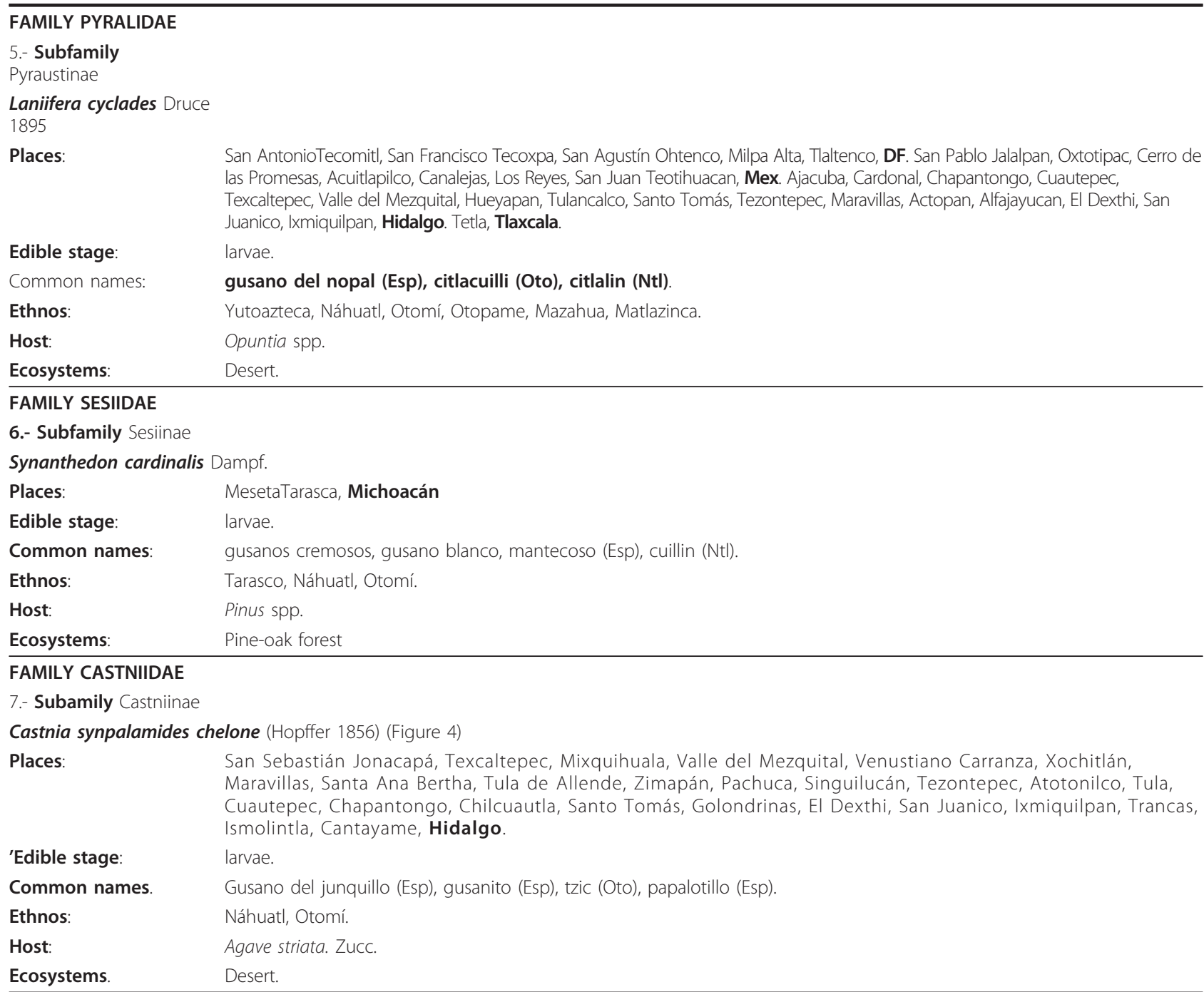

FAMILY GEOMETRIDAE

8.- Subfamily: Ennominae

Acronyctodes mexicanaria (Walker 1860)

Places: $\quad$ Topilejo, Santa Ana Tlacotenco, San Lorenzo Tlacoyucan, San Juan Tepenahuac, San Pedro Actopan, Milpa Alta, DF.

Edible stage: $\quad$ larvae and pupae.

Common names: $\quad$ Temictli (Oto), Tetatamachiuhqui (Ntl).

Ethnos: Yutoazteca, Náhuatl, Otomí.

Host: Budleia spp.

Ecosystems. Savanna, arid tropical scrub, Oak-Forest.

9.- Subfamily: Ennominae

Panthera pardalaria Hübner 1823

Places: San Simón Tlatlahuilpa Tlaxcala, Torres del Potrero DF.

Edible stage: larvae

Common names: Huitzitsi (Oto).

Ethnos: Náhuatl, Otomí, Yutoazteca.

Host: Family Graminae

Ecosystems: $\quad$ Cultures of graminae 
Table 1 TAXONOMY (Continued)

\section{FAMILY HESPERIIDAE (Figura 5) \\ 10.- Subfamily Megathyminae}

Aegiale hesperiaris (Walker 1856) (Figure 5)

Places:

San Pedro Atocpan, San Salvador Cuahtenco, San Jerónimo Miacatlán, Santa Ana Tlacotenco, San Bartolo Xicomulco, San Lorenzo Tlacoyucan, San Agustín Ohtenco, San Pablo Oztotepec, San Antonio Tecomitl, San Francisco Tecoxpa, San Juan Tepenahuac, Milpa Alta DF. San Juan Zitlaltépetl, Santa María Jajalpan, San Bartolo Morelos, Huixquilucán, Lomas de Guadalupe, Cuautitlán Izcalli, Cuautitlán de Romero Rubio, Aculco, Almoloya de Juárez, Santiago Tianguistenco, Almoloya del Río, Atlacomulco, Ixtlahuaca, Jalatlaco, Jilotepec, Zumpango, Los Reyes, Ozumba, San Pablo Jalalpan, Toluca, Villa del Carbón, Villa Nicolás Romero, Otumba, Arroyo Zarco, Santiago Tilapa, El Oro, Aguatepec, San Pedro de los Baños, San Mateo, Mex. Guanajuato, Guanajuato. Santo Tomás, Huichapán, Chilcuatla, San Nicolás Atexcoco, Maravillas, Zimapán, Cuautepec, Jacalá, Pinalito, Ixmiquilpan, Pozuelos, Cieneguillas, Ajacuba, Apan, Atotonilco el Grande, Atotonilco de Tula, Texcaltepec, Tlaxcoapan, Tulancalco, Valle del Mezquital, Xochitlán, Tulancingo, Durango, el Cajón, Pachuquilla, San Miguel Regla, Metztitlán Mixquihuala, Molango, Pachuca, Singuilucan, Tula de Allende, Trancas, Ismolintla, Venustiano Carranza, Venta de Guadalupe, San Sebastián Jonacapa, Tinaco, Santa Ana Bertha, Tezontepec, Chapantongo, Tepetitlán, El Sauce, Ixtaltepec, Alfajayucan, El Dexthi, San Juanico, Ixmiquilpan, Hidalgo. Tlalpujahua, San Pedro Tarimbaro Michoacán, Santa María Nduayaco, Santiago Apoala, Oaxaca, Ciudad Serdán, Acatlán de Osorio, Puebla. San Juan del Río, Querétaro, Calpulalpan, Cuapixtla, Huamantla, Ixtacuixtla, Nativitas, San Pablo del Monte, Tetla, Totolac, Xicoténcatl, Mariano Matamoros, Tlaxcala. Perote, Naolinco, Veracruz. Fresnillo, Zacatecas.

Edible stage:

Common name:

Ethnos:

Host:

Ecosystems. larvae.

gusano blanco del maguey (Esp), gusanito del maguey (Esp), meocuiles (Nt), meocuilines (Ntl), ticoco andabi (Mix), zat (Zap), yabi (My), guinches (Maz), Nnchaama (Tar), Chucugame (Mat), huitzipapalotl (Ntl), papálotl (Ntl).

Yutoazteca, Náhuatl, Otomí, Otopame, Mazahua, Matlazinca, Tarasco, Zapoteco, Mixteco, Totonaco, Huasteco, Maya.

Agave atrovirens Karw., A. salmiana, Otto ex Salm, A. mapisaga Trel, A. lehmanni. Jacobi, A. maximiliana, Baker. A. americana.

Desert, pine-oak forest.

11.- Subfamily Pyrginae

Achlyodes pallida (Felder, 1869)

Places. San Pablo Huixtepec, Oaxaca, Tenejapa, Chiapas

Edible stage: Larvae

Common names: $\quad$ chiáhuitl (Mix), saltadora (Esp), papalotl (Ntl).

Ethnos: $\quad$ Zapoteco, Mixteco, Mixe, Populaca, Chatino, Chinanteco, Mazateco, Zoque, Trique, Huave, Tojolabal, Maya, Tzotzil, Tzeltal, Chol, Lacandón.

Host: Citrus aurantium L., C. sinensis

Ecosystems: $\quad$ Cultures of lucerne and maize.

FAMILY PAPILIONIDAE

12.- Subfamily Papilioninae

Protographium philolaus philolaus (Boisduval, 1836) (Figure 6)

Places:

Caezim, Yucatán

Edible stage: larvae.

Common name: $\quad$ Tlilizic (My).

Ethnos: Maya.

Host: Annona cherimola, A. diversifolia, A. purpurea, A. reticulata; Desmopsis bibracteata and Sapranthus spp.

Ecosystems. Tropical decidous forest.

13.- Subfamily Papilioninae

Pterourus multicaudata multicaudata (Kirby, 1884)

Places: Santiago Tezontlale, Hidalgo

Edible stage: adult.

Common name: $\quad$ mariposa de colores (Esp), xochiquetzal (Nt).

Ethnos: Náhuatl, Otomí

Host: $\quad$ Fraxinus sp., Prunus persica L., P. serotina

capuli.

Ecosystems.

Decidous forest, Oak forest

\section{FAMILY PIERIDAE}

14.- Subamily Coliadinae

Phoebis agarithe agarithe (Boisduval) 1836 (Figure 7)

Places:

Caezim, Yucatán 
Table 1 TAXONOMY (Continued)

\begin{tabular}{|c|c|}
\hline Edible stage: & larvae. \\
\hline Common names: & gusano pinto (Esp), pintillo (Esp), clac (My), xicalpapálotl (Ntl). \\
\hline Ethnos: & Maya. \\
\hline Host: & Cassia tomentosa L., Inga sp. \\
\hline Ecosystems: & Tropical decidous forest \\
\hline \multicolumn{2}{|c|}{ 15.- Subfamily Coliadinae } \\
\hline \multicolumn{2}{|c|}{ Phoebis philea philea (Linnaeus 1763) } \\
\hline Places: & Celaya, Irapuato, Guanajuato \\
\hline Edible stage: & larvae. \\
\hline Common name: & Ocuil (Ntl). \\
\hline Ethnos: & Otomi, Tarasco. \\
\hline Host: & Casia tomentosa L.; Senna spp. \\
\hline Ecosystems. & "Acahual" \\
\hline \multicolumn{2}{|c|}{ 16.- Subfamily Coliadinae } \\
\hline \multicolumn{2}{|c|}{ Phoebis sennae marcellina (Cramer 1779) } \\
\hline Places: & San Juan Tezompa, Villa Guerrero, Mex. \\
\hline Edible stage: & larvae \\
\hline Common names: & Tlaxic (Oto), Papalotli (Ntl), Tzauhqui (Maz). \\
\hline Ethnos: & Otopame, Mazahua, Matlazinca. \\
\hline Host: & Cassia sp.; Senna, Inga \\
\hline Ecosystems: & "Acahual" \\
\hline \multicolumn{2}{|c|}{ 17.- Subfamily Coliadinae } \\
\hline \multicolumn{2}{|c|}{ Eurema salome jamapa (Reakirt 1866) } \\
\hline Places: & Tempoal de Sánchez, Veracruz. \\
\hline Edible stage: & larvae \\
\hline Common names: & Papalotl (Nt) \\
\hline Ethnos: & Totonaco, Huasteco. \\
\hline Host: & Picramnia sp, Diphysa robinoides Benth \\
\hline Ecosystems: & "Acahual" \\
\hline \multicolumn{2}{|c|}{ 18.- Subfamily Pierinae } \\
\hline \multicolumn{2}{|c|}{ Eucheira socialis socialis (Westwood 1834) (Figure 8). } \\
\hline Places: & $\begin{array}{l}\text { San Cristóbal de las Casas, Chiapas. Caborachi y sudeste de Chihuahua. San Antonio Tecomitl, San Francisco Tecoxpa, } \\
\text { San Mateo, San Lorenzo Tlacoandula, San Agustín Ohtenco, Santa Ana Tlacotengo, San Jerónimo Miacatlán, Milpa Alta, } \\
\text { Tlaltenco, Topilejo, DF. La Michilía, Durango, Donato Guerra, Villa Victoria, Cerro de las Promesas, Oxtotipac, San Pablo } \\
\text { Jalalpan, Valle de Bravo, Villa de Allende, Mex. Chacoalcingo, Guerrero. Santo Tomás, Valle del Mezquital, Atlapexco, } \\
\text { Huasca, Durango, Tecocomulco, Actopán, Maravillas, Tezontepec, Hidalgo. Tlalpujahua, Cerro del Gallo, San Pedro } \\
\text { Tarímbaro, Michoacán, Nochixtlán, Santa María Nduayaco, Santa María de la Asunción, Tlaxiaco, Oaxaca. Ciudad Serdán, } \\
\text { Chignahupan, Tetela de Ocampo, Puebla. Tetla, Tlaxcala. Orizaba, Veracruz. }\end{array}$ \\
\hline Edible stage: & larvae. \\
\hline Common name: & mariposa del madroño (Esp), gusano del madroño (Esp), gusano verde de la mixteca (Esp), Nnchaama (Tar). \\
\hline Ethnos: & $\begin{array}{l}\text { Maya, Tzotzil, Tzeltal, Chol, Lacandon, Tojolabal Tarahumara, Yutoazteca, Náhuatl, Otomí, Tepehuano, Otopame, Mazahua, } \\
\text { Matlazinca, Tlapaneco, Amuzgo, Tarasco, Zapoteco, Mixteco, Mixe, Popoluca, Chatino, Chinanteco, Mazateco, Zoque, } \\
\text { Trique, Huave, Totonaco, Huasteco. }\end{array}$ \\
\hline Host: & Arbutus xalapensis H.B.K.. A. arizonica, A. glandulosa and A. macrophylla \\
\hline Ecosystems: & Pine-Oak forest, Arid tropical scrub. \\
\hline \multicolumn{2}{|c|}{ 19.- Subfamily Pierinae } \\
\hline \multicolumn{2}{|c|}{ Eucheria socialis westwoodi (Beutelspacher 1984). } \\
\hline Places: & La Michilía, Durango. \\
\hline Edible stage: & larvae. \\
\hline Common name: & mariposa del madroño (Esp), gusano del madroño (Esp), gusano verde de la mixteca (Esp), Nnchaama (Tar). \\
\hline Ethnos: & Tepehuano, Tarahumara. \\
\hline Host: & Arbutus sp. \\
\hline Ecosystems. & Pine-Oak forest, Arid tropical scrub. \\
\hline
\end{tabular}


Table 1 TAXONOMY (Continued)

\begin{tabular}{|c|c|}
\hline \multicolumn{2}{|c|}{ 20.- Subfamily Pierinae } \\
\hline \multicolumn{2}{|c|}{ Catasticta teutila teutila Doubleday 1847 (Figures 9 and 10). } \\
\hline Places: & $\begin{array}{l}\text { San Francisco Tlalnepantla, Xochimilco, Santa Ana Tlacotenco Milpa Alta, Topilejo, DF. Juchitepec, Mex. Santa María } \\
\text { Nduayaco, Santiago Apoala, Oaxaca. }\end{array}$ \\
\hline Edible stage: & larvae, pupae. \\
\hline Common name: & Mariposa del tejocote (Esp), Tlilpapálotl (Ntl). \\
\hline Ethnos: & $\begin{array}{l}\text { Yutoazteca, Náhuatl, Otomí, Otopame, Mazahua, Matlazinca, Zapoteco, Mixteco, Mixe, Popoluca, Chatino, Chinanteco, } \\
\text { Mazateco, Zoque, Trique, Huave. }\end{array}$ \\
\hline Host: & Viscum álbum L. Phoradendron velutinum (DC) Nutt. \\
\hline Ecosystems: & Pine-oak forest, Tropical evergreen forest and Tropical decudous forest. \\
\hline \multicolumn{2}{|c|}{ 21.- Subamily Pierinae } \\
\hline \multicolumn{2}{|c|}{ Catasticta flisa flisa (Herrich-Schäffer 1853) } \\
\hline Places: & $\begin{array}{l}\text { San Francisco Tlalnepantla, Xochimilco, Milpa } \\
\text { Alta DF. }\end{array}$ \\
\hline Edible stage: & larvae. \\
\hline Common name: & Mariposa del tejocote (Esp), Nixtapapalotl (Nt) \\
\hline Ethnos: & Yutoazteca, Náhuatl, Otomí. \\
\hline Host: & Phoradendron velutinum (DC) Nutt. \\
\hline Ecosystems: & Pine-oak forest, Tropical evergreen forest and thorn forest. \\
\hline \multicolumn{2}{|c|}{ 22.- Subfamily Pierinae } \\
\hline \multicolumn{2}{|c|}{ Catasticta nimbice nimbice (Boisduval, 1836). } \\
\hline Places: & San Francisco Tlalnepantla, Xochimilco, Milpa Alta, DF. \\
\hline Edible stage: & larvae. \\
\hline Common name: & Papalotl (Ntl), Papalotontle (Oto). \\
\hline Ethnos: & Yutoazteca, Náhuatl, Otomí. \\
\hline Host: & Phoradendron velutinum (DC) Nutt. \\
\hline Ecosystems: & Pine-oak forest, Tropical evergreen forest and thorn forest. \\
\hline \multicolumn{2}{|c|}{ 23.- Subfamily Pierinae } \\
\hline \multicolumn{2}{|c|}{ Pontia protodice (Boisduval \& Leconte 1829). } \\
\hline Places: & Valle de México. \\
\hline Edible stage: & Larvae. \\
\hline Common names: & Tilpapalotl (Nt), \\
\hline Ethnos: & Otopame, Mazahua, Matlazinca. \\
\hline Host: & Brassica oleracea $\mathrm{L}$. \\
\hline Ecosystems. & Cultures of lucerne, cabbage, and Oak Forest. \\
\hline \multicolumn{2}{|c|}{ 24.- Subfamily Pierinae } \\
\hline \multicolumn{2}{|c|}{ Leptophobia aripa elodia (Boisduval, 1836) } \\
\hline Places: & Valle de México. \\
\hline Edible stage: & larvae. \\
\hline Common names: & Chiahuitl (Oto) \\
\hline Ethnos: & Otopame, Mazahua, Matlazinca. \\
\hline Host: & Brassica rapa L., Lepidium sativum L., Tropaeolum majus L. \\
\hline Ecosystems: & Cultures of cabbage, cauliflower and broccoli. \\
\hline \multicolumn{2}{|c|}{ 25.- Subfamily Nymphalinae } \\
\hline \multicolumn{2}{|c|}{ Vanessa annabella (Field 1971) } \\
\hline Places: & Santo Tomás, Hidalgo. \\
\hline Edible stage: & larvae and pupae. \\
\hline Common name: & gusano (Esp), Papalotepito (Ntl), Quiloculin (Oto). \\
\hline Ethnos: & Náhuatl, Otomí. \\
\hline Host: & Malva sp., Althaea rosea L. \\
\hline Ecosystems: & Pine-oak forest, arid tropical scrub. \\
\hline
\end{tabular}


Table 1 TAXONOMY (Continued)

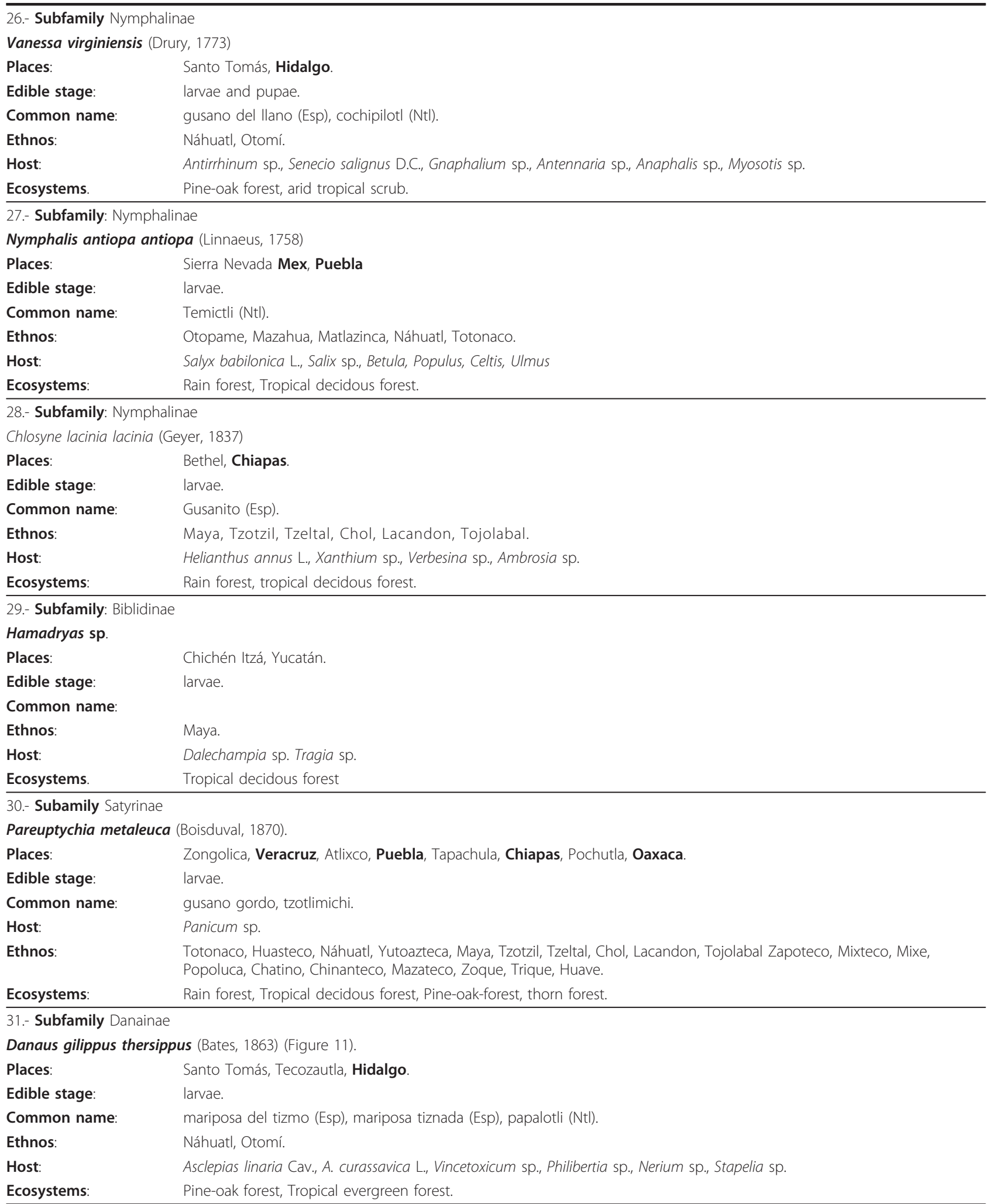


Table 1 TAXONOMY (Continued)

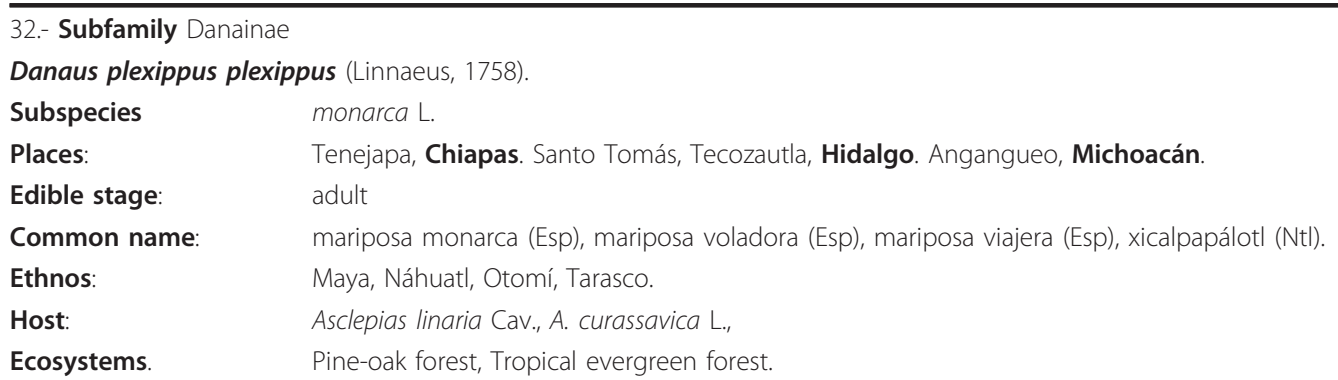

\section{FAMILY BOMBYCIDAE}

33.- Subfamily: Bombycinae

Bombyx mori (Linnaeus, 1758).

Yosotato, Oaxaca.

Edible stage

larvae.

Common name:

gusano de seda (Esp), sedaocuilin (Ntl), tzauhquiocuilin (Nt)).

Ethnos:

Zapoteco, Mixteco, Mixe, Popoluca, Chatino, Chinanteco, Mazateco, Zoque, Trique, Huave.

Host:

Morus rubra var rubra $L$.

Ecosystems.

Cloud forest, Rain forest.

\section{FAMILY LASIOCAMPIDAE}

34.- Subfamily Lasiocampinae

Eutachyptera psidii (Sallé, 1857)

$\begin{array}{ll}\text { Places: } & \text { Laguna Atezca, Molango, Hidalgo. } \\ \text { Edible stage: } & \text { larvae } \\ \text { Common name: } & \text { Mecta'che (Ntl), tecilli (Oto). } \\ \text { Ethnos: } & \text { Náhuatl, Otomí. } \\ \text { Host: } & \text { Psidiun guajaba L. } \\ \text { Ecosystems: } & \text { Cloud forest }\end{array}$

FAMILY SATURNIIDAE

35.- Subfamily Arsenurinae

Arsenura armida (Cramer, 1779) (Figure 12).

Places:

Molango, Hidalgo. Jamiltepec, Oaxaca. Cuezalán, Santiago Yancuitlalpan, Coatepec de Matamoros, Acatlán de Osorio Puebla, Santiago Tuxtla, Los Tuxtlas, el Bajío, Chocamán, Ixcohuapa, Veracruz.

Edible stage: larvae.

Common name: $\quad$ Cuecla (Nt), serpiente de mil cabezas (Esp), culebron (Esp), chonocuile (Mzt), cuetano (Mix), pochocuil (Zap), Zapala (Mx), tilpapálotl (Nt), Tecocoz (Pop). $\begin{array}{ll}\text { Ethnos: } & \text { Náhuatl, Otomí, Zapoteco, Mixteco, Mixe, Popoluca, Chatino, Chinanteco, Mazateco, Zoque, Trique, Huave, Totonaco, } \\ & \text { Huasteco. }\end{array}$

Host: $\quad$ Ceiba pentandra L. (Pochote), Chorisia sp. Heliocarpus appendiculatus Turcz.

Ecosystems: $\quad$ Tropical decidous forest, Tropical evergreen forest, Pine oak-forest.

36.- Subfamily Arserurinae

Arsenura polyodonta (Jordan, 1911).

Places: $\quad$ Atzitzihuacán, Atlixco, Puebla.

Edible stage: larvae.

Common name: $\quad$ cuecla (Ntl), zats (Tot), cuitlame (Maz), gusano del jonote (Esp).

Ethnos: Náhuatl, Totonaco.

Host: Malvaceae, Tiliaceae, Chorisia sp.

Ecosystems: Pine-oak forest.

37.- Subfamily Arserurinae

Caio championi (Druce, 1886).

Places:

sur de Veracruz.

Edible stage:

larvae.

Common names:

Cuillicuatl (Ntl)

Ethnos:

Nahuatl, Totonaco, Huasteco. 
Table 1 TAXONOMY (Continued)

\begin{tabular}{|c|c|}
\hline Host: & Bombacopsis sp., Chorisia sp., Tilia sp. \\
\hline Ecosystems. & Tropical decidous forest, Tropical evergreen forest. \\
\hline \multicolumn{2}{|c|}{ 38.- Subfamily Arserurinae } \\
\hline \multicolumn{2}{|c|}{ Caio richardsoni (Druce, 1890). } \\
\hline Places: & Cahuaré Chiapas. Chapantongo Hidalgo. \\
\hline Edible stage: & Larvae. \\
\hline Common names: & Guano oscuro (Esp), ocul (Ntl), culli (Oto). \\
\hline Ethnos: & Maya, Tzotzil, Tzeltal, Chol, Lacandon, Tojolabal, Náhuatl, Otomí. \\
\hline Host. & Chorisia sp., Ceiba pentandra L. \\
\hline Ecosystems: & Mesquite-grassland, Arid tropical scrub, Tropical decidous forest, Tropical evergreen forest. \\
\hline \multicolumn{2}{|c|}{ 39.- Subfamily Ceratocampinae } \\
\hline \multicolumn{2}{|c|}{ Eacles aff. ormondei yucatanensis (Lemaire, 1988) } \\
\hline Places: & Zongolica, Ixcohuapa Veracruz \\
\hline Edible stage: & Larvae \\
\hline Common names: & Tlecocoz (Oto). \\
\hline Ethnos: & Náhuatl, Yutoazteca. Otomí. \\
\hline Host: & Quercus sp., Rhus sp. \\
\hline Ecosystems: & Cloud forest, Oak-forest. \\
\hline
\end{tabular}

40.- Subfamily Ceratocampinae

Eacles sp. Hübner

\begin{tabular}{ll} 
Places: & Puerto Morelos, Quintana Roo. \\
Edible stage: & larvae. \\
Common name: & gusanito (Esp), xixicalticon (My). \\
Ethnos: & Maya. \\
Host: & Malvaceae, Melastomataceae. \\
Ecosystems: & Tropical evergreen forest. \\
\hline
\end{tabular}

41.- Subfamily Hemileucinae

Hemileuca sp. (Walker, 1855)

Places: $\quad$ Zinacantepec, Mercado de Toluca, Almoloya de Juárez, Calixtlahuaca, Villa Victoria, Mex.

Edible stage: larvae.

Common name: $\quad$ zacamiches (Maz).

Ethnos: Otopame, Mazahua, Matlazinca.

Host: Salix sp., Fagaceae, Leguminosae, Rosaceae.

Ecosystems: Pine-Oak forest.

42.- Subfamily Hemileucinae

Hylesia frigida Schaus, 1911.

Places: $\quad$ Navenchauc, Zinacantán, Coapilla, Chiapas. Santa María Nduayaco, Santiago Apoala, Asunción Nochixtlán, Oaxaca.

Edible stage: $\quad$ larvae.

Common name: $\quad$ Nn-chúm (Tzo), calocuillin (Nt), caliocuillin (Tze).

Ethnos: Maya, Tzotzil, Tzeltal, Chol, Lacandon, Tojolabal, Zapoteco, Mixteco, Mixe, Popolaca, Chatino, Chinanteco, Mazateco, Zoque, Trique, Huave.

Host: $\quad$ Pinus sp., Bursera sp.. Anacardiaceae, Lauraceae, Melastomataceae.

Ecosystems: Pine-oak forest, Pine-Forest, Cloud forest, Deciduos Forest.

43.- Subfamily Hemileucinae

Hylesia coinopus Dyar, 1913.

Places: Cahuaré,

Chiapas.

Edible stage: larvae.

Common name: $\quad$ mariposa de hilo grande (Esp), ciulicuactl (Tze).

Ethnos: Maya, Tzotzil, Tzeltal, Chol, Lacandon, Tojolabal.

Host: Pinus sp., Bursera sp., Anacardiaceae, Lauraceae, Melastomataceae.

Ecosystems: Tropical decidous forest. 
Table 1 TAXONOMY (Continued)

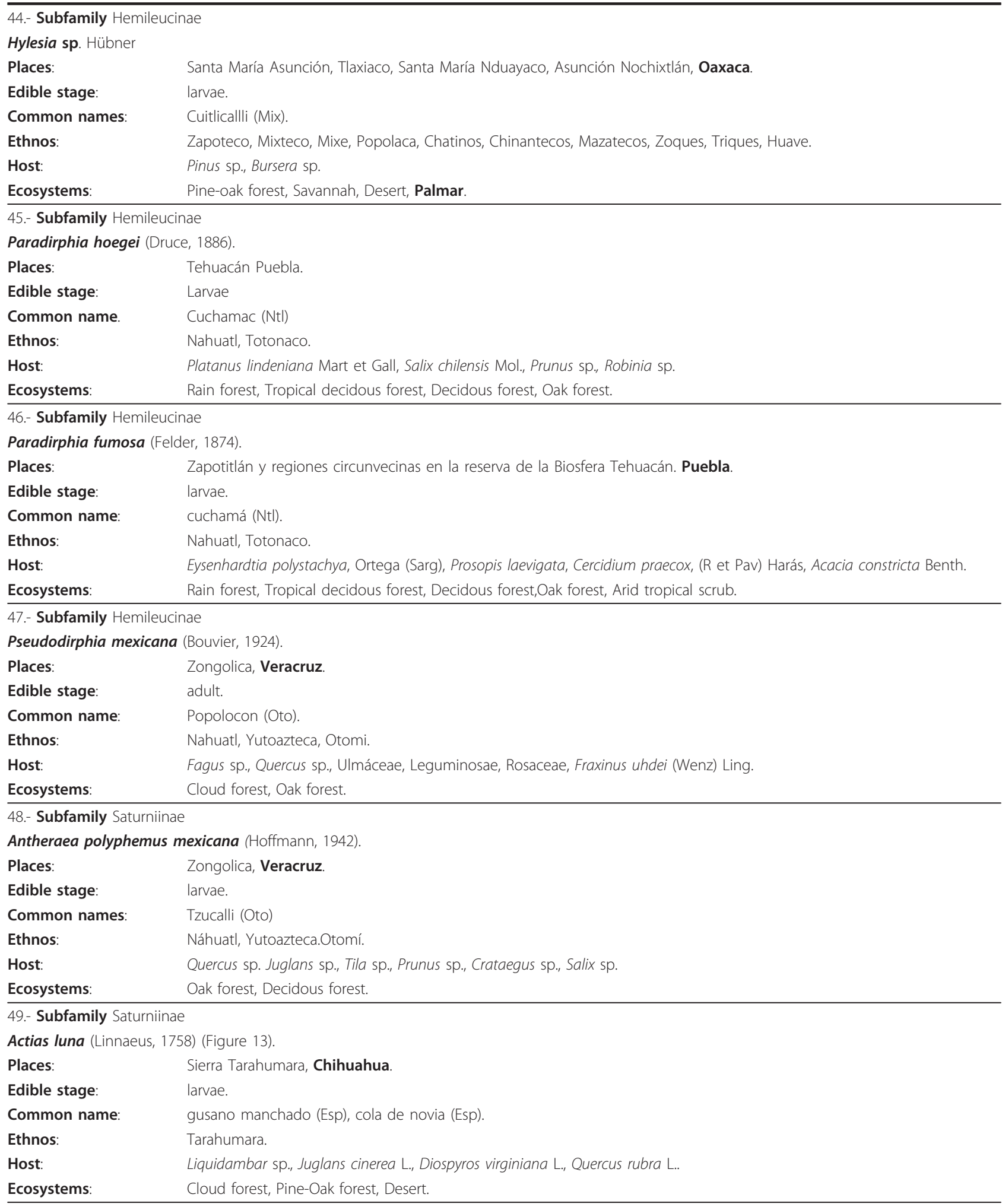

50.- Subfamily Saturniinae

Actias truncatipennis (Sonthonnax 1899)

Places: Sierra Tarahumara, Chihuahua

Edible stage: larvae. 
Table 1 TAXONOMY (Continued)

\begin{tabular}{|c|c|}
\hline Common name: & gusano gordo (Esp), papalotli (Ntl). \\
\hline Ethnos: & Tarahumara. \\
\hline Host: & Liquidambar sp, Juglandaceae \\
\hline Ecosystems: & Cloud forest. \\
\hline \multicolumn{2}{|c|}{ FAMILY SPHINGIDAE } \\
\hline \multicolumn{2}{|c|}{ 51.- Subfamily Macroglossinae } \\
\hline \multicolumn{2}{|c|}{ Pachylia ficus (Linnaeus, 1758) } \\
\hline Places: & Motozintla, Chiapas. \\
\hline Edible stage: & larvae. \\
\hline Common name: & gusano (Esp). \\
\hline Ethnos: & Maya, Tzotzil, Tzeltal, Chol, Lacandon, Tojolabal. \\
\hline Host: & Ficus cookii Stand. \\
\hline Ecosystems: & Forest of Poeppigia procera Presl. \\
\hline \multicolumn{2}{|c|}{ 52.- Subfamily Sphinginae } \\
\hline \multicolumn{2}{|c|}{ Cocytius antaeus (Cramer, 1777) (Figure 14). } \\
\hline Places: & Cahuaré, Chiapas, Tixtla, Guerrero. \\
\hline Edible stage: & larvae. \\
\hline Common name: & gusano cornudo (Esp), gusano del cuerno (Esp). \\
\hline Ethnos: & Maya, Tzotzil, Tzeltal, Chol, Lacandon, Tojolabal, Náhuatl, Tlapaneco, Amuzgo. \\
\hline Host: & Anona spp. \\
\hline Ecosystems: & Thorn forest. \\
\hline \multicolumn{2}{|c|}{ 53.- Subfamily Sphinginae } \\
\hline \multicolumn{2}{|c|}{ Manduca sexta (Linnaeus, 1763). } \\
\hline Places: & Cahuaré Chiapas, Ixcohuapa, Veracruz \\
\hline Edible stage: & larvae, adult. \\
\hline Common name: & gusano del cuerno (Esp). \\
\hline Ethnos: & Maya, Náhuatl, Yutoazteca, Otomí. \\
\hline Host: & Nicotiana tabacum L. \\
\hline Ecosystems: & Cloud forest, Oak forest. \\
\hline
\end{tabular}

54.- Subfamily Sphinginae

\section{Manduca sp.}

Places: Ixcohuapa, Veracruz.

Edible stage: larvae, adult.

Common name: $\quad$ gusano grande verde (Esp).

Ethnos: Náhuatl, Yutoazteca, Otomí.

Host: Nicotiana tabacum L.

Ecosystems: Cloud forest.

\section{FAMILY NOCTUIDAE}

55.- Subfamily Calpinaenae

Ascalapha odorata (Linneaus, 1758) (Figure 15).

Places: $\quad$ Tuxtla Gutiérrez, Bochil, Frontera, Bethel, Selva Lacandona, Chiapas. Coyoacán, DF. Tixtla, El Potrero, Zacazonapan, Colotlipa, Mezcantepec, Quechultenango, Chilpancingo, Guerrero. Teotitlán del Camino, Oaxaca. San Juan Epatlán, Izúcar de Matamoros, Atlixco, Ajalpan, Coatepec de Matamoros, Puebla. Puerto Morelos, Quintana Roo.

Edible stage: larvae.

Common name: $\quad$ mariposa del muerto (Esp), cuetla (Nt)), cuetlacuahuetl (Ntl), pochocuiles (Oto), cuetano (Mix).

Ethnos: Maya, Tzotzil, Tzeltal, Chol, Lacandon, Tojolabal, Yutoazteca, Náhuatl, Otomí, Tlapaneco, Amuzgo, Zapoteco, Mixteco, Mixe, Popoluca, Chatino, Chinanteco, Mazateco, Zoque, Trique, Huave, Totonaco.

Host: Melastomataceae

Ecosystems: Thorn forest, Tropical evergreen forest, Rain forest, Tropical decidous forest.

56.- Subfamily: Calpinaenae

Ascalapha agarista Cramer, 1777.

Places: Chilpancingo, Guerrero 
Table 1 TAXONOMY (Continued)

\begin{tabular}{|c|c|}
\hline Edible stage: & larvae. \\
\hline Common name: & mariposa de la muerte (Esp), mariposa del muerto (Esp). \\
\hline Ethnos: & Náhuatl, Tlapaneco, Amuzgo. \\
\hline Host: & Melastomataceae. \\
\hline Ecosystems. & Tropical decidous forest \\
\hline \multicolumn{2}{|c|}{ 57.- Subfamily: Agaristinae } \\
\hline \multicolumn{2}{|c|}{ Gerra sevorsa (Grote, 1882). } \\
\hline Places: & Pedregal de San Ángel, DF, San Miguel Regla, Hidalgo. Misantla, Veracruz. \\
\hline Edible stage: & larvae. \\
\hline Common name: & Gusano del maíz (Esp) \\
\hline Ethnos: & Yutoazteca, Náhuatl, Otomí, Totonaco, Huasteco. \\
\hline Host: & Unknown \\
\hline Ecosystems. & Cloud forest, Pine-Oak forest, and Arid tropical scrub. \\
\hline \multicolumn{2}{|c|}{ 58.- Subfamilly Calpinae } \\
\hline \multicolumn{2}{|c|}{ Latebraria amphipyroides (Guenée, 1852) } \\
\hline Places: & $\begin{array}{l}\text { Frontera, Echeverria, Argovia, las Cañitas, Selva Lacandona, Ixtapa, Bethel, Independencia, Frontera, Chiapas. San Pedro } \\
\text { Atocpan, San Salvador Cuahtenco, San Jerónimo Miacatlán, Santa Ana Tlacotenco, San Bartolo Xicomulco, San Lorenzo } \\
\text { Tlacoyucan, San Agustín Ohtenco, San Pablo Oztotepec, San Antonio Tecomitl, San Francisco Tecoxpa, San Juan } \\
\text { Tepenahuac, Milpa Alta, DF. Huejutla de Reyes, Atlapexco, Durango, Santo Tomás, Xochitlán, Chilcuautla, Romantla, } \\
\text { Hidalgo, Santa María Nduayaco, Santiago Apoala, Huajuapan de Léon, Yosotato, Puerto Escondido, Oaxaca. Izúcar de } \\
\text { Matamoros, Tehuitzingo, Santa Inés Ahuatempan, Puebla. Orizaba, Ixtapaluka, Chocaman, Veracruz. }\end{array}$ \\
\hline Edible stage: & larvae. \\
\hline Common name: & Cuetla (Ntl), Cuetlmami (Oto), culebra gorda (Esp), culebra cornuda (Esp). \\
\hline Ethnos: & $\begin{array}{l}\text { Maya, Tzotzil, Tzeltal, Chol, Lacandon, Tojolabal, Yutoazteca, Náhuatl, Otomí, Totonaco, Huasteco, Mixe, Popoluca, Chatino, } \\
\text { Chinanteco, Mazateco, Zoque, Trique, Huave. }\end{array}$ \\
\hline Host. & Ipomea intrapilosa Rose \\
\hline Ecosystems: & Rain forest, Tropical decidous forest, Pine-oak Forest, Arid tropical scrub, Pine-Forest. \\
\hline \multicolumn{2}{|c|}{ 59.- Subfamily Calpinae } \\
\hline \multicolumn{2}{|c|}{ Thysania agrippina (Cramer, 1776) } \\
\hline Places: & Tenejapa, Chiapas. \\
\hline Edible stage: & larvae. \\
\hline Common name: & mariposa águila (Esp), mariposon (Esp), mazacuata (Tzo). \\
\hline Ethnos: & Maya, Tzotzil, Tzeltal, Chol, Lacandón, Tojolabal. \\
\hline Host: & Unknown \\
\hline Ecosystems. & Rain forest, Tropical evergreen forest. \\
\hline \multicolumn{2}{|c|}{ 60.- Subfamily Heliotinae } \\
\hline \multicolumn{2}{|c|}{ Helicoverpa zea (Boddie, 1850). } \\
\hline Places: & $\begin{array}{l}\text { San Pedro Atocpan, San Salvador Cuahtenco, San Jerónimo Miacatlán, Santa Ana Tlacotenco, San Bartolo Xicomulco, San } \\
\text { Lorenzo Tlacoyucan, San Agustín Ohtenco, San Pablo Oztotepec, San Antonio Tecomitl, San Francisco Tecoxpa, San Juan } \\
\text { Tepenahuac, Milpa Alta, Pedregal de San Angel, DF. Villa de Allende, Polotitlán, Jilotepec, San Francisco Chimalpa, San } \\
\text { José Tezompa, Temamatla, Santiago Tilapa, Tequixquiac, Mex. Quechultenango, Mezcantepec, Chilpancingo, Guerrero. } \\
\text { Atlapexco, Chilcuautla, Valle del Mezquital, Durango, Santo Tomás, Xochitlán, Molango, San Miguel Regla, Tlaxcoapan, El } \\
\text { Dexthi, San Juanico Ixmiquilpan, Hidalgo. Santa María Nduayaco, Santiago Apoala, Oaxaca. Tenancingo, Tetla, Tlaxcala, } \\
\text { Ixcohuapa, Veracruz. }\end{array}$ \\
\hline Edible stage: & larvae. \\
\hline Common name: & gusano del maíz (Esp), gusano de la milpa (Esp). \\
\hline Ethnos: & $\begin{array}{l}\text { Yutoazteca, Náhuatl, Otomí, Otopame, Mazahua, Matlazinca, Zapoteco, Mixteco, Mixe, Popoluca, Chatino, Chinanteco, } \\
\text { Mazateco, Zoque, Trique, Huave, Totonaco, Huasteco. }\end{array}$ \\
\hline Host. & Zea mays L. \\
\hline Ecosystems: & Cultures of maize mixed with beans, geen beans and lucerne. \\
\hline
\end{tabular}

61.- Subfamily Xyleninae

Spodoptera exigua (Hübner, 1808).

$\begin{array}{ll}\text { Places: } & \text { Zapotitlán, Tláhuac, DF. } \\ \text { Edible stage: } & \text { larvae. } \\ \text { Common name: } & \text { gusano soldado (Esp). }\end{array}$


Table 1 TAXONOMY (Continued)

\begin{tabular}{|c|c|}
\hline Ethnos: & Yutoazteca, Náhuatl, Otomí. \\
\hline Host: & Zea mays L. \\
\hline Ecosystems. & Cultures of maize. \\
\hline \multicolumn{2}{|c|}{ 62.- Subfamily Xyleninae } \\
\hline \multicolumn{2}{|c|}{ Spodoptera frugiperda (Smith, 1797). } \\
\hline Places: & $\begin{array}{l}\text { San Pedro Atocpan, San Salvador Cuahtenco, San Jerónimo Miacatlán, Santa Ana Tlacotenco, San Bartolo Xicomulco, San } \\
\text { Lorenzo Tlacoyucan, San Agustín Ohtenco, San Pablo Oztotepec, San Antonio Tecomitl, San Francisco Tecoxpa, San Juan } \\
\text { Tepenahuac, Milpa Alta, DF. Villa de Allende, Polotitlán, San José Tezompa, Santa Anita, Temamatla, Tequixquiac, Mex. } \\
\text { Tlaxcoapan, Tulancalco, Hidalgo. Tetla, Tlaxcala. }\end{array}$ \\
\hline Edible stage: & Larvae. \\
\hline Common name: & gusano elotero (Esp). \\
\hline Ethnos: & Yutoazteca, Náhuatl, Otomí, Otopame, Mazahua, Matlazinca. \\
\hline Host: & Zea mays L. \\
\hline Ecosystems. & Cultures of maize. \\
\hline \multicolumn{2}{|c|}{ 63.- Subfamily Xyleninae } \\
\hline \multicolumn{2}{|c|}{ Spodoptera sp. } \\
\hline Places: & Milpa Alta, DF. \\
\hline Edible stage: & larvae. \\
\hline Common name; & gusano soldado (Esp). \\
\hline Ethnos: & Yutoazteca, Náhuatl, Otomí. \\
\hline Host: & Zea mays $L$. \\
\hline Ecosystems: & Culture of maize mixed with beans, greenbeans and lucerne. \\
\hline
\end{tabular}
FAMILY ARCTIIDAE

64. - Subfamily: Arctiinae

Pelochyta cervina (Edwards, 1884).

$\begin{array}{ll}\text { Places: } & \text { Zongolica, Veracruz } \\ \text { Edible stage: } & \text { adult. }\end{array}$

Common Name:

$\begin{array}{ll}\text { Ethnos: } & \text { Náhuatl, Yutoazteca, Otomí. } \\ \text { Host: } & \text { Scientific name unknown, common name "cucharilla real". } \\ \text { Ecosystems: } & \text { Rain forest and Tropical decidous forest. }\end{array}$

65.- Subfamily: Arctiinae

Elysius superba (Druce, 1884) (Figure 16)

Places: Zongolica, Veracruz

Edible stage: larvae.

Common name: $\quad$ gusano del palo mulato (Esp).

Ethnos: Náhuatl, Yutoazteca, Otomí.

Host: Bursera simaruba Sarg., Ficus sp.

Ecosystems: Rain forest and Tropical decidous forest.

66.- Subfamily Arctiinae

Amastus ochreaceator Walker, 1865) (Figure 17)

Places: Zongolica, Veracruz.

Edible stage: larvae.

Common name: $\quad$ gusano de los palos (Esp), xicaltetecon (Ntl).

Ethnos: Náhuatl, Yutoazteca, Otomí.

Host: Inga jinicuil Schl.

Ecosystems. Rain forest and Tropical decidous forest.

67.- Subfamily: Arctiinae

Estigmene acrea (Drury, 1773) (Figure 18)

Places: Zongolica, Veracruz.

Edible stage: adult.

Common name: $\quad$ oruga salina (Esp). 
Table 1 TAXONOMY (Continued)

\begin{tabular}{ll}
\hline Ethnos: & Náhuatl, Yutoazteca, Otomí. \\
Host: & Phaseolus vulgaris, Mimosa af. pigra L. \\
Ecosystems. & Rain forest, Tropical decidous forest. \\
\hline
\end{tabular}

Languages: Español (Esp); Matlazinca (Mat); Maya (My); Mazahua (Maz); Mazateco (Mzt); Mixe (Mx); Mixteco (Mix); Nahuatl (Ntl); Otomi (Oto); Popoluca (Pop);

Tarasco (Tar); Totonaco (Tot); Tzetzal (Tze); Tzotzil (Tzo); Xochimilca (Xoc); Zapoteco (Zap).

for helping people to have a good health and satisfaction of energy and proteins requirements.

\section{Marketing and Gastronomy}

The trade of Lepidoptera larvae still persist being sold in markets in several areas of the country and even at the capital, as the red and white agave worms. In five forks restaurants of Mexico City. These species are in great demand, in large part due to their exquisite flavor, though the eating of these worms is also an ancestral tradition and a signal of power in diverse sectors of the population. Due to the high demand for these species, some sellers of them have special refrigerators for freezing and storing them. In this way, they can offer and prepare them at high prices after the collecting season.

There are other genera, such as Phassus, for which people search laboriously, it has a very similar flavor to chicken, while Laniifera cyclades "nopal worm" has a flavor of a fried potato. In the humid-tropical areas, "cuetla" and "cuecla" larvae, corresponding to Latebraria amphipyroides and Arsenura armida are pickled to give the larvae a flavor similar to herring, while the Spodoptera spp. is similar to that of corn (Table 4).

Unfortunately, these organisms are the subject of massive gathering in several of the States of Mexico, where they are profusely eaten. Thus, they could be in danger of extinction, due to the lack of rules regarding their collection, distribution and commercialization [31].

\section{Cultures and Proto-cultures}

In Mexico, some Lepidoptera are raised. Leptophobia aripa elodia, Pieris brassicae also the silk worm Bombyx mori in the States of Oaxaca and San Luis Potosí. Their industrial management is widely known, because of

Table 2 Families and species number

\begin{tabular}{cccccc}
\hline Family & Species & Family & Species & Family & Species \\
\hline Hepialidae & 3 & Hesperiidae & 2 & Saturniidae & 16 \\
Cossidae & 1 & Papilionidae & 2 & Sphingidae & 4 \\
Pyralidae & 1 & Pieridae & 11 & Noctuidae & 9 \\
Sesiidae & 1 & Nymphalidae & 8 & Arctiidae & 4 \\
Castniidae & 1 & Bombycidae & 1 & Total of & 67 \\
& 2 & Lasiocampidae & 1 & & \\
Geometridae & 2 & &
\end{tabular}

their economical importance in China, Japan, India, France, and Italy.

Eucheira socialis socialis the green worm of the Huasteca region widely distributed has larvae that are located inside a secreted silk enclosure of papyraceous consistency. The larvae hang on the branches of Arbutus xalapensis, feeding on young leaves [32]. In some parts of it, people make a "protoculture" that maintain on the edges of their house roof. They hang at least three silk enclosures (each bag contains only one sex), if they do that, the protoculture will survive. In the zone of the Oaxaqueña Mixteca, specially in the towns of Santa María Nduayaco and Santiago Apoala, this species disappeared due to the great degree of consumption; this species has since been reintroduced from Durango and Mexico states [33].

Some ethnobiological studies have been conducted on the red and white agave worms [34]. We investigate on their biology, ecology, and ethology, to increase their production by optimization of their culture particularly in Santo Tomàs, Montecillo, and Apan in the State of Hidalgo and in the laboratory $[35,36]$ with this we developed the biotechnology that would allow their culture on a greater scale. In fact, this technology for such cultures can be purchased in the Intelectual Property Direction of the UNAM [37]. Also, studies have been conducted to characterize the development of the larvae of the red agave worm [38].

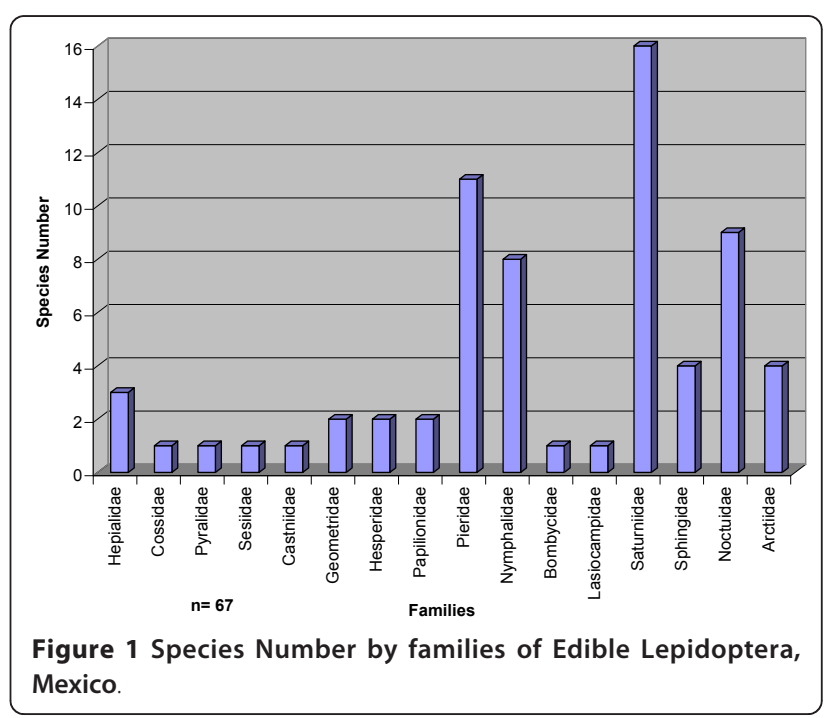




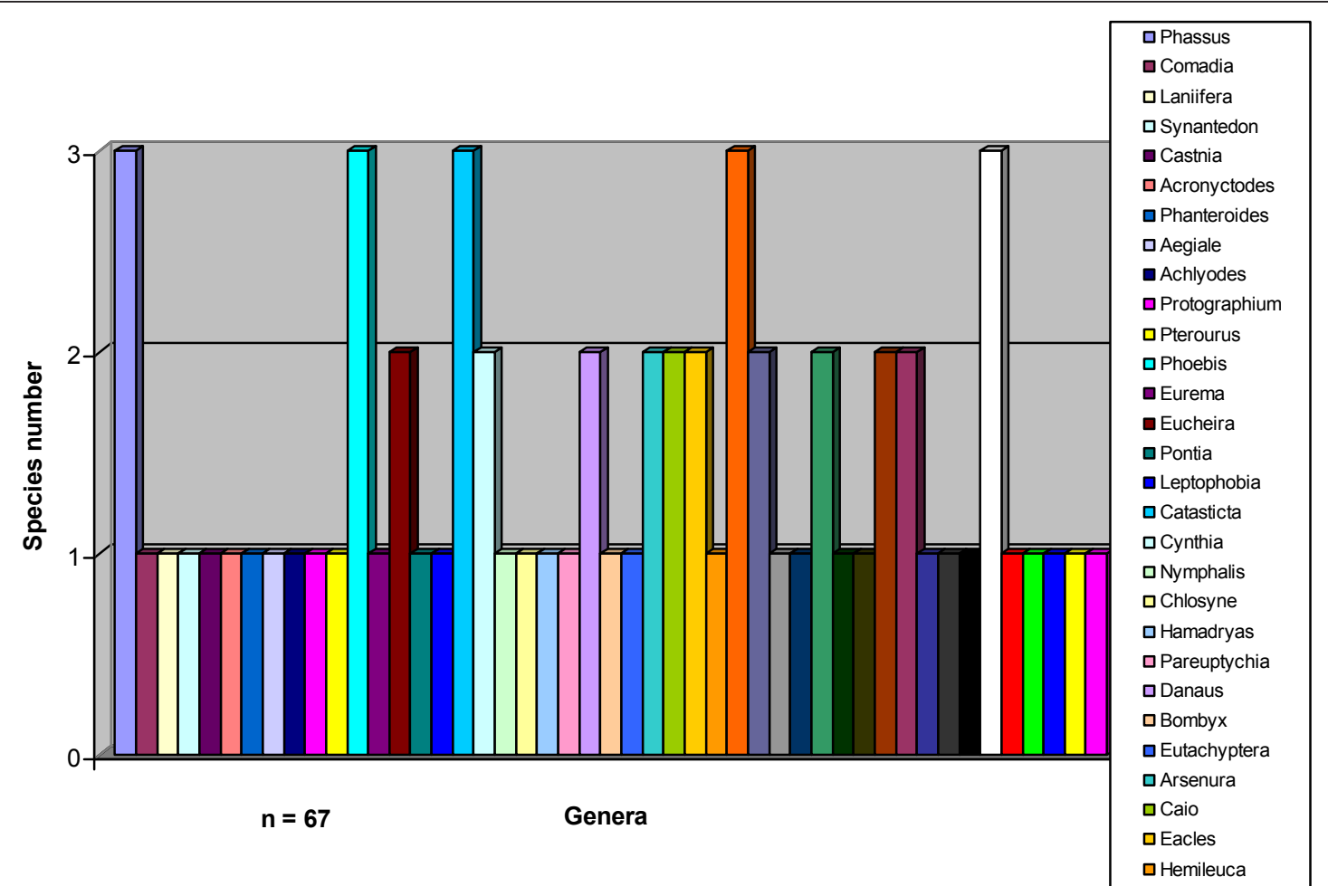

Figure 2 Species number of edible Lepidoptera in Mexico

\section{Sustainable Management}

The management and conservation of the species Paradirphia fumosa has been implemented in Mexico at the Biosphere Reserve of Tehuacán-Cuicatlán [39,40].

In this aspect we must also recognize the deep knowledge that indigenous people all over the world have, as they possess $90 \%$ of the planet's germplasm $[41,42]$ because they have maintained a high degree of sustainability with the majority of their resources.

\section{Biomass obtention}

These species are recollected by their abundance, because in some ones their recollection could be measure in tons [43] as it happens today with Ascalapha odorata or Latebraria amphypirioides stored in big cotton sacs of $50 \mathrm{~kg}$ and offered in market day or in a "tianguis" (market of little towns). Other species they could also sell alive while they are inside a little sac that they build in silk form their nests, as it is in gregarious

Table 3 Genus and species number

\begin{tabular}{|c|c|c|c|c|c|c|c|}
\hline Genus & Species & Genus & Species & Genus & Species. & Genus & Species \\
\hline Phassus & 3 & Eucheira & 2 & Caio & 2 & Helicoverpa & 1 \\
\hline Comadia & 1 & Pontia & 1 & Eacles & 2 & Latebraria & 1 \\
\hline Laniifera & 1 & Leptophobia & 1 & Hemileuca & 1 & Spodoptera & 3 \\
\hline Synantedon & 1 & Catasticta & 3 & Hylesia & 3 & Thysania & 1 \\
\hline Castnia (Synpalamides) & 1 & Cynthia & 2 & Paradirphia & 2 & Pelochyta & 1 \\
\hline Acronyctodes & 1 & Nymphalis & 1 & Pseudodirphia & 1 & Elisus & 1 \\
\hline Phanteroides & 1 & Chlosyne & 1 & Antheraea & 1 & Amastus & 1 \\
\hline Aegiale & 1 & Hamadryas & 1 & Actias & 2 & Estigmene & 1 \\
\hline Achlyodes & 1 & Pareuptychia & 1 & Pachylia & 1 & Total of species & 67 \\
\hline Protoghraphium & 1 & Danaus & 2 & Cocytius & 1 & & \\
\hline Pterourus & 1 & Bombyx & 1 & Manduca & 2 & & \\
\hline Phoebis & 3 & Eutachyptera & 1 & Ascalapha & 2 & & \\
\hline Eurema & 1 & Arsenura & 2 & Gerra & 1 & & \\
\hline
\end{tabular}


Table 4 Genus and Species most consummed in México

\begin{tabular}{|c|c|c|c|c|c|}
\hline Family & Genus & Species & Family & Genus & Species \\
\hline \multirow[t]{3}{*}{ Hepialidae } & Phassus & Trajesa & Saturniidae & Arsenura & polyodonta \\
\hline & $P$ & triangularus & & Caio & richardsoni \\
\hline & $P$. & sp. & & Hylesia & coinopus \\
\hline Cossidae & Comadia & redtenbacheri & & H. & frigida \\
\hline Castniidae & Castnia (Synpalamides) & chelone & & $H$. & sp. \\
\hline Pyralidae & Laniifera & cylades & Noctuidae & Ascalapha & odorata \\
\hline Geometridae & Acronyctodes & mexicanaria & & A. & agarista \\
\hline Hesperidae & Aegiale & hesperiaris & & Helicoverpa & zea \\
\hline \multirow[t]{3}{*}{ Pieridae } & Catasticta & teutila teutila & & Latebraria & amphiphyrioides \\
\hline & C. & flisa flisa & & Spodoptera & frugiperda \\
\hline & Eucheira & socialis socialis & & S. & exigua \\
\hline Saturniidae & Arsenura & armida & & & \\
\hline
\end{tabular}
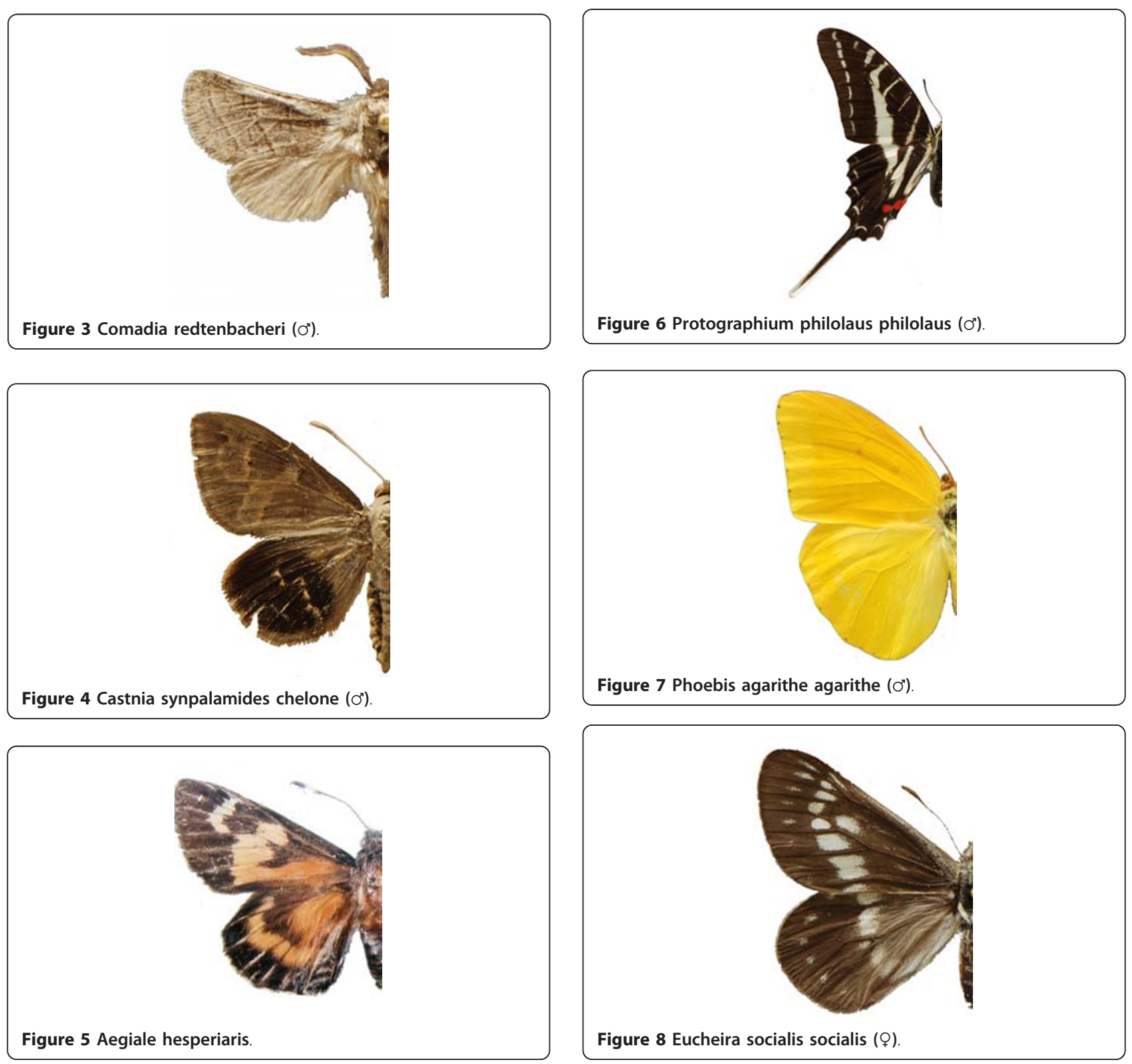

Figure 8 Eucheira socialis socialis $(\%)$. 

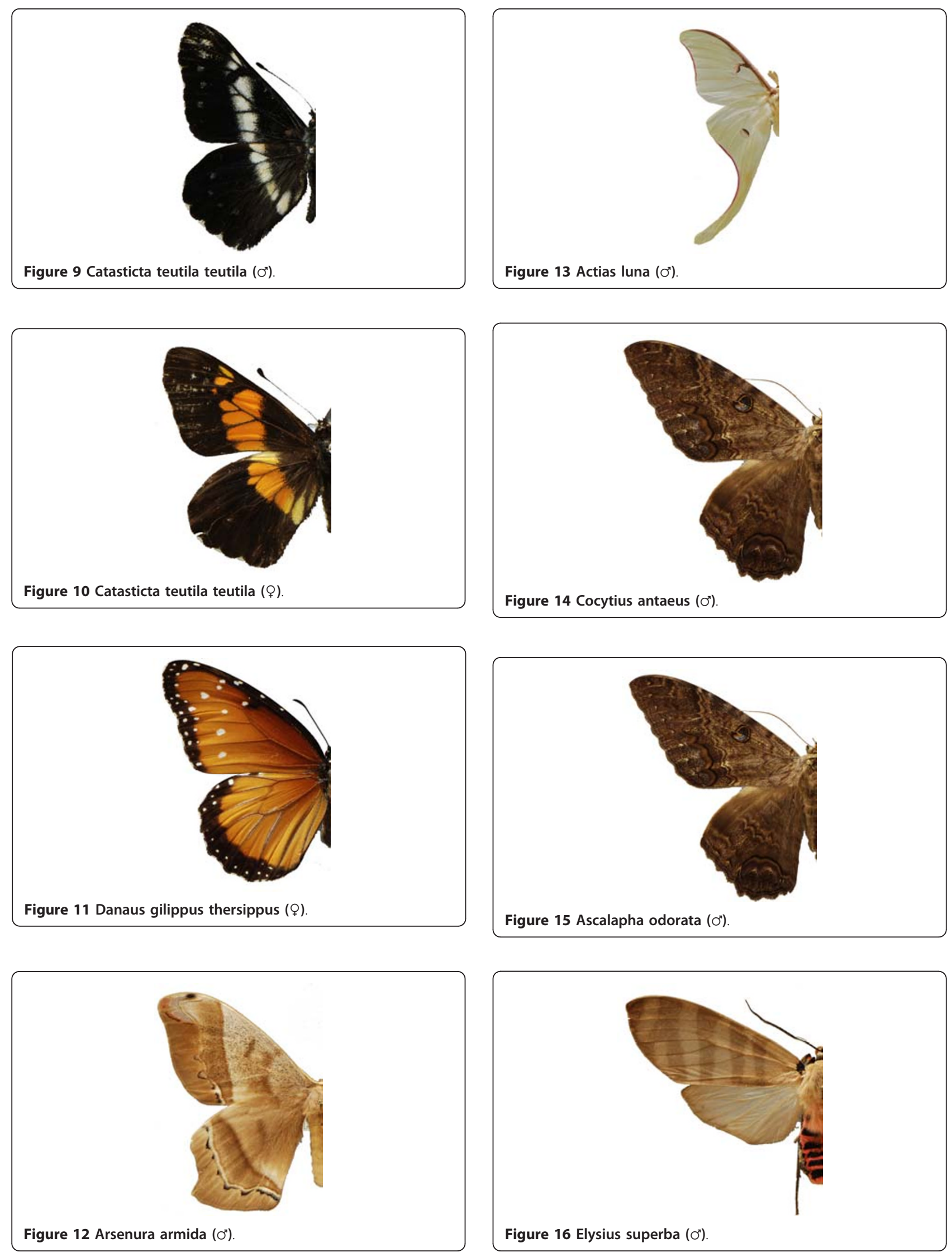


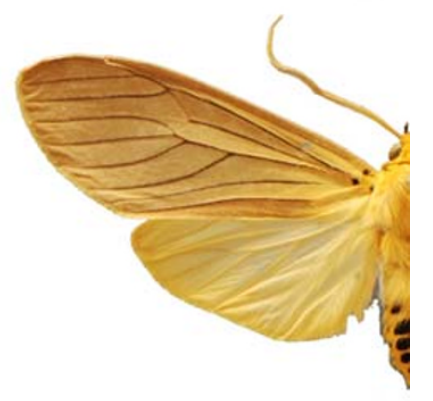

Figure 17 Amastus ochreaceator (o')

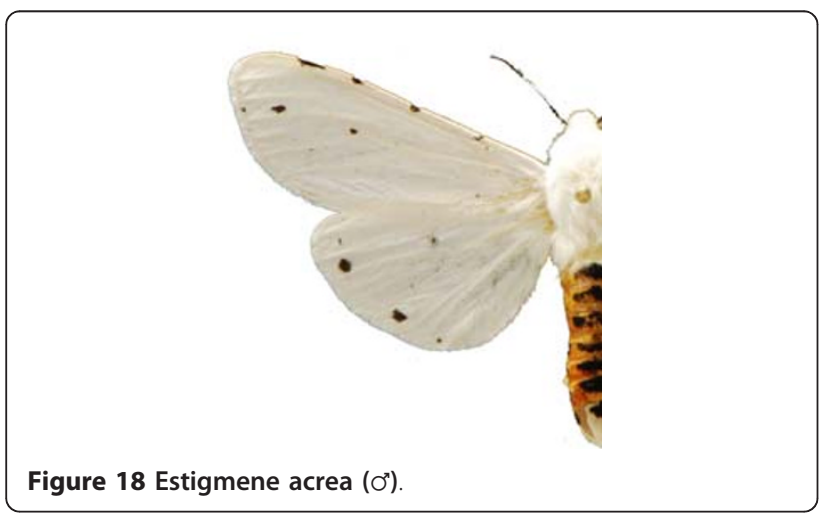

species, Eucheira socialis or Hylesia frigida. Other species are captured by the use of net as is Phoebis agarithe, or the "monarch butterfly" (Danaus plexipus plexipus, D. gilippus thersippus) or Pterourus multicaudata multicaudata where people do not eat the larvae because this makes the heart stop, but adults. In other species they could be found many individuals together inside their hosts as in Comadia redtenbacheri or Laniifera cyclades, with the help of a hunting knife or even collected the prepupa digging the soil around.

\section{Preparation}

Generally is the larvae that are eaten. They are prepared roasted with salt, and in populations with a higher economic purchase they are fried with oil or lard joining always pepper, salt, in tortillas (maize crepes). They could be boiled and roasted in a "pan" or justly fried with salt and pepper, wormseed leaves. Also, boiled split into longitudinale axis, mixed with oil. Boiled, drained and stuffed with fresh cheese, or it could be with tuna or cooked with eggs, like an omelette. Also in a pie accompanied or mixed with rice, as are the shrimps in the "paella" transfering to rice a very special and good flavour.

They could be preserved in brine, and cutted into small pieces in the same way as used crouttons or bacon.
The flavours are really peculiar and it is dificult to compare with something known, but we can said they varied from light delicious flavours to strong and different unkown flavours.

Peasants qualified them as a very good and nutritious "worms", ipure vitamine!, to refer to the quantity of proteins they lodged.

\section{Trade and Marketing Nets}

Many species are traded and sell by fits or sardine cans. Another way to sell them is already boiled in salt water or preserved in brine. Generally, they are not offer in fixed places in trough the market, but street sellers are walking in different corridors of these, asking people to buy them, by example Comadia redtenbacheri, Aegiale hesperiaris, Arsenura armida, Ascalapha odorata, and Latebraria amphipyroides are sold on the plaza days, market days, in ambulant markets or on roadsides and even in the Mexico City market. They are offered in big plastic boxes or baskets and are measured in tuna or sardine cans, or frequently in "cazuelitas" (little ceramic dishes of different sizes). Some of the recorded edible Lepidoptera thus clearly constitute an important part of the nutrition and economy of the Mexican people $[33,34]$, particularly for the indigenous collectors, middlemen, distributors, salesmen, and restaurant owners. In addition, canned white agave worms are exported to the United States and Canada by the enterprises Clemente Jacques and Elan, S.A., and thus generate foreign income for Mexico. This worm has been sold for $\$ 250.00$ USD per kilogram (2006), which is ten times more expensive than a fish or beef fillet. The exported cans cost $\$ 50.00$ Canadian dollars; these cans contain only 5 or 6 larvae of the last or penultimate larval stage.

Other species have also been commercialized, such as "zacamiches", Hemileuca sp. at Toluca market, "gusanillo", Phassus triangularis, and P. trajesa at different markets in the Veracruz State and "cuchama" (Paradirphia fumosa) in Tehuacán, Puebla.

A little more than $10 \%$ (8 species) of edible Lepidoptera larvae are commercialized, but many more species are sold in the adult stage at very high prices.

Some African species in the larval stage are preserved by pickling and are then exported to European cities. In Paris, France, for example, they are offered in the market of La Rue Moufetard in the Latin neighborhood. These are sold in huge fiber baskets, and can be seen in the street markets in several localities for sale on different days of the week. They are mostly bought by immigrants in those countries [32].

Some examples of Edible Lepidoptera of Mexico are Figures 3, 4,5, 6, 7, 8, 9, 10, 11, 12, 13, 14, 15, 16, 17 and 18. 


\section{Acknowledgements}

We are very grateful to Dr. Carlos Beutelspacher, Dr. Manuel Balcàzar and Biól. Lucio Rivera by their help in the taxonomical subject.

\section{Author details}

'Instituto de Biología, UNAM, Apartado Postal 70-153, 04510, México DF, México. ${ }^{2}$ Facultad de Ciencias Biológicas y Agropecuarias UV, Córdoba Veracruz, México.

\section{Authors' contributions}

All authors read and approved the final manuscript. JRE, Author responsable for the project and publication, writting, editing of the manuscript. JMPM, Collect, preliminary preparation of the manuscript, editing, labels, catalogs, literature review. AlV, Assembly and identification of species of Lepidoptera. ILT, Collect of different species in diverses states of Mexico. HOR, Identification of host plants. VHMC. Writting and formatting the manuscript, references research on internet and all computer work.

\section{Authors' information}

Dra. Julieta Ramos-Elorduy: has the highest position as researcher at the Institute of Biology of the National University of Mexico and professor of postgraduate courses at the Faculty of Science of the same University. She have 104 scientific publications and four books published. 1153 cites of its publications and 1316 on internet. She lead 152 thesis and publish 289 divulgation articles.

M.en C. José Manuel Pino Moreno: Biologist and M.Sc. by the Faculty of Science of the UNAM (National University of Mexico), Academic Technical of the Institute of Biology and Professor of the Faculty of Sciences both of the UNAM. He has published like co-author several articles about antropoentomophagy and medicinal insects and one book. Adolfo Ibarra Vázquez. Technical Lepidoptera collection of the Institute of Biology of the UNAM.

M en C. Ivonne Landero Torres. Biologist and M.Sc. by the University of Veracruz, Urban Management and Promotion, Professor of the Faculty of Biology, Cordoba. She has published like co-author several articles about anthropoentophagy.

Héctor Oliva-Rivera. Biologist and M.Scy by the University of Veracruz, Plant Taxonomy Professor of the Faculty of Biology, Cordoba.

Biologist Victor Hugo Martínez Camacho by the Faculty of Science of the UNAM (National University of Mexico), he has published like co-author one chapter of book and several articles of edible insects.

\section{Competing interests}

The authors declare that they have no competing interests.

Received: 28 July 2010 Accepted: 6 January 2011

Published: 6 January 2011

\section{References}

1. Dufour DL: Insects as food: A case of study from the Northwest Amazon. Am Anthropol 1987, 89:383-397.

2. Ramos-Elorduy J, Conconi M: Edible insects of the world. Abstracts Fourth Int. Congress of Ethnobiology Lucknow, India; 1994, 311.

3. Ramos-Elorduy J: Creepy Crawly Cuisine Rochester, VT: Park Street Press; 1998.

4. Ramos-Elorduy J: Energy supplied by edible insects from Mexico and their nutritional and ecological importance. Ecol Food Nutr 2008, 47:280-297.

5. Hogue CL: Latin American Insects and Entomology California: University of California Press, Berkeley; 1993.

6. Mbata KJ: Traditional use of arthropods in Zambia. Food Insects Newsletters 1995, 8:1, 5-7.

7. Holden S: Edible caterpillars. A potential agroforestry resource? Food Insects Newsletter 1991, 4:4-5.

8. Ramos-Elorduy J, Pino MJM, Martínez CVH: Base de datos de los insectos comestibles de México UNIBIO-IBUNAM; 2008.

9. Costa-Neto EM: In Manual de Etnoentomologia. Volume IV. España: Zaragoza; 2002.

10. Balcazar LM, Wolfe LK: Cladistics of the Ceratocampinae (Lepidoptera: Saturniidae). Tropical Lepidoptera 1997, 8:1-53.
11. Beutelspacher BCR: Mariposas Diurnas del Valle de México. México Ediciones Científicas L.P.M.M; 1980

12. Beutelspacher BCR: Catálogo de la Colección Roberto Müller (Lepidoptera: Heterocera) del Museo de Historia Natural de la Ciudad de México México: Cuadernos de Biología, UNAM; 1992.

13. Heppner JB, Classification of Lepidoptera: Part 1, "Introduction". Holartic Lepidoptera 1998, 5:1-148.

14. Hoffmann CC: Catálogo sistemático y zoogeográfico de los lepidópteros mexicanos. Soc. Mex. de Lepidopterología Publicaciones Especiales 1976, 1:1-214.

15. Llorente BJ, Oñate OL, Martínez AL, Vargas Fl: Papilionidae y Pieridae de México: Distribución Geográfica e llustración México: UNAM Fac. de Ciencias; 1997.

16. Stone ES: Food plants of world saturniidae. Memoirs of the Lepidopterists Society 1991, 4:1-185.

17. Warren AD: Hesperoidea (Lepidoptera). In Biodiversidad, taxonomía y biogeografía de artrópodos de México: hacia una sintesis de su conocimiento. Volume II. Edited by: Llorente J, y González E. México: UNAM; 2000:535-580.

18. De Vries P: In The butterflies of Costa Rica and their natural history. Volume II. Princeton: Princeton University Press; 1997.

19. Martínez M: Catalogo de nombres vulgares y cientificos de plantas mexicanas México: Fondo de Cultura Económica, México; 1979.

20. Rzedowski J: Vegetación de México Limusa, México; 1978.

21. Bergier E: Peuples Entomophages et Insectes Comestibles France: Rullière Frères, Avignon; 1941.

22. Taylor R: Butterflies in my Stomach or: Insects in Human Nutrition Santa Barbara California: Woodbridge Press Publishing Co; 1975

23. Silow CA: Edible and other insects of Mid-western Zambia. Sudies in Ethno-entomology II. Occ Pap V Inst Allm Jamforand Etnogr Uppsala, Sweden: Almqvist \& Wiksell; 1976, 64-69.

24. Malaisse F, Parent G: Les chenilles comestibles du Shaba Méridionale (Zaire). Nat Belges 1980, 61(1):2-24.

25. Latham P: Edible caterpillars and their food plants in the Bas Congo, Democratic Republic of Congo. Botanic Gardens Conservations News 1999, 3(3):40-42.

26. Banjo $A D$, Lawal $O A$, Songonuga $E A$ : The nutritional value of fourteen species of edible insects in southwestern Nigeria. Afr J Biotechnol 2006, 5:298-301.

27. Oliveira J, Passos de Carvalho J, Bruno de Sousa R, Sinao M: The nutritional value of four species of insects consumed in Angola. Ecol Food Nutr 1976, 5:91-97.

28. Wen L-Z: Principle and Application of Edible Entomology Press of Hunan Science and Technology. Changsha. China; 1998.

29. Mitsuhashi J: Edible Insects in the World Tokio: Kokin Shoin; 1984, (In Japanese)

30. Paoletti MG, Dufour DL, Cerda H, Torres F, Pizzoferrato L, Pimentel D: The importance of leaf and litter-feeding invertebrates as source of animal protein for the Amazonian Amerindians. Proc Roy Soc LondB Bio Sci 2000, 267:2247-2252.

31. Ramos-Elorduy J, Pino MJM, Conconi M: Ausencia de una reglamentación y normalización de la explotación y comercialización de insectos comestibles en México. Folia Entomol Mexic 2006, 45:291-318.

32. Ramos-Elorduy J: Importance of edible insects in the nutrition and economy of the people of the rural areas in México. Ecol Food Nutr 1997, 36:347-366.

33. Ramos-Elorduy J, Pino MJM, Ladrón de GO, Lagunes J: Edible insects of Oaxaca State Mexico and their nutritive value. J Food Compos Anal 1997, 10:142-157.

34. Manzano MJ: Estudio etnobiológico del gusano de maguey (Aegiale (Acentrocneme) hesperiaris Walker, Cossus redtenbacheri Hammerschmidth y Scyphophorus acupunctatus Gyll.) en el municipio de Apan Hidalgo Tesis Prof. UNAM; 1989.

35. Cisneros BY: Estudios biológicos acerca del gusano blanco de maguey (Aegiale (Acentrocneme) hesperiaris W. Lepidoptera-Megathymidae). Thesis Prof., UNAM, Faculty of Science, Department of Biology; 1988.

36. Aguilar RA: Contribución al conocimiento bioecotológico del gusano blanco de maguey (Aegiale (Acentrocneme) hesperiaris W.). Thesis Prof. UNAM, Faculty of Science, Department of Biology; 1995.

37. Ramos-Elorduy J: Tecnología para la producción de gusano blanco de maguey Aegiale hesperiaris W. (Lepidoptera-Megathymiidae) Incubadora de Empresas UNAM; 2000. 
38. Hernández LRA, Llanderal CC, Nieto Hernández RL, Castillo ME, Valdés CJ: Determinación de instares larvales del gusano rojo de maguey. Entomología Mexicana 2006, V:289-292.

39. Rosas AML: Estudios sobre la biología y comportamiento del gusano rojo de maguey Comadia redtenbacheri H. (Lepidoptera-Cossidae) y su producción sustentable. Thesis M.S. Benemérita Universidad Autónoma de Puebla; 2008.

40. Velásquez SI, González LLA, Porras MA: El gusano cuchamá: fuente alternativa y sustentable de alimento y empleo en Zapotitlán Salinas, Estado de Puebla, México. XVII Conferencia Internacional "Estrategias de desarrollo y alternativas para América y el Caribe"; 2006, 1-39.

41. Posey DA: O conhecimiento entomológico Kayapó. etnometodologia e sistema cultural. Anuario Antropológico 1983, 81:109-121.

42. Posey DA: The application of Ethnobiology in the conservation of dwindling natural resource: lost knowledge or options for the survival of the planet. In Ethnobiology: Implications and Applications. Proceedings of the first international Congress of Ethnobiology 1988. Edited by: Posey DA, Leslie Overal W. Belém, Brazil; 1990:47-60.

43. Ramos-Elorduy J: Insects a hopeful food. In Ecological Implications of Minilivestock (Potential of Insects, Rodents, Frogs and Snails). Edited by: M Paoletti G. Oxford IBH Publishing; 2005:263-291.

doi:10.1186/1746-4269-7-2

Cite this article as: Ramos-Elorduy et al: Edible Lepidoptera in Mexico: Geographic distribution, ethnicity, economic and nutritional importance for rural people. Journal of Ethnobiology and Ethnomedicine 2011 7:2.

\section{Submit your next manuscript to BioMed Central} and take full advantage of:

- Convenient online submission

- Thorough peer review

- No space constraints or color figure charges

- Immediate publication on acceptance

- Inclusion in PubMed, CAS, Scopus and Google Scholar

- Research which is freely available for redistribution

Submit your manuscript at www.biomedcentral.com/submit
C Biomed Central 